\title{
Usefulness of Post-labeling Delay for the Original Assessment of Bright Vessel Appearance by Arterial Spin Labeling
}

Taishi Chiba, ${ }^{1}$ Hayato Suzuki, ${ }^{2}$ Suguru Yamaguchi, ${ }^{2}$ and Katsuhiro Nishino ${ }^{2}$

Objective: This study was performed to clarify the differences in blood flow strength, blood vessel diameter, and postlabeling delay (PLD) by physical experiments, and to examine whether bright vessel appearance (BVA) can be observed by arterial spin labeling (ASL).

Methods: We introduced simulated blood flow $(25 \mathrm{~cm} / \mathrm{sec}, 12.5 \mathrm{~cm} / \mathrm{sec})$ using a specially made phantom of fixed tubes in a plastic container. At each speed, we scanned at several points of PLD using ASL imaging. We measured the signal in the tube to obtain a signal intensity $(\mathrm{SI})$. We revised the $\mathrm{T} 1$ level from the $\mathrm{SI}$ and obtained $\mathrm{SI}_{\text {blood. }}$. We used $\mathrm{SI}_{\text {tissue }}$ with normal perfusion measured from obtained clinical images by ASL and compared it with $\mathrm{SI}_{\text {blood }}$.

Results: In tubes with a narrow inner diameter, the signal slightly decreased. SI also decreased under slow flow compared with fast flow. At each flow rate, $\mathrm{SI}_{\text {blood }}$ significantly exceeded $\mathrm{SI}_{\text {tissue }}$.

Conclusion: PLD distinguishes spin in brain tissue from $1525 \mathrm{msec}$ to $2525 \mathrm{msec}$, and it can be observed. As spin signal decreases when the flow rate is slow, attention is necessary for observation. Assessment at PLD1525-2525 msec where normal perfusion was obtained suggested that BVA can be observed.

Keywords > MRI, arterial spin labeling, post-labeling delay, acute ischemic stroke, bright vessel appearance

\section{Introduction}

Several studies have reported the efficacy of endovascular treatment in acute ischemic stroke (AIS) patients with over 6 hours after the onset through satisfactory

${ }^{1}$ Department of Radiology, Kakunodate General Hospital, Senboku, Akita, Japan

${ }^{2}$ Department of Neurosurgery, Kakunodate General Hospital, Senboku, Akita, Japan

Received: December 3, 2019; Accepted: April 24, 2020 Corresponding author: Chiba Taishi. Department of Radiology, Kakunodate General Hospital, 3, Iwase, Kakunodate, Senboku, Akita 014-0394, Japan

Email: renji6481@yahoo.co.jp

*The abstract of this study was presented at The 34th Annual Meeting of The Japanese Society for Neuroendovascular Therapy held on November 22nd-24th, 2018 in Sendai, Miyagi, Japan.

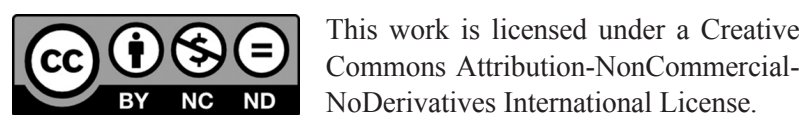

(C)2020 The Japanese Society for Neuroendovascular Therapy case selection for mechanical thrombectomy. ${ }^{1,2)}$ These randomized controlled trials also suggested the importance of perfusion image-based condition assessment. The importance of MRI, which non-invasively provides perfusion images, in AIS treatment has been emphasized.

A method of obtaining non-contrast-enhanced perfusion images using MRI, arterial spin labeling (ASL), has been routinely applied in the field of brain disease. It is also useful for AIS patients. ${ }^{3-5)}$ In the process of ASL, a specific waiting time until brain tissue perfusion, known as post-labeling delay (PLD), is established using water molecules (spin) in blood as a tracer, and perfusion images are obtained.

When cerebrovascular occlusion or stenosis is present, spin perfusion to the brain tissue is delayed, and the residual spin is detected as high signal intensity (SI) in blood vessels. This can be observed on original images, suggesting the presence of a collateral pathway or site of occlusion in AIS patients. This is termed bright vessel appearance (BVA). ${ }^{6-8)}$

However, on ASL, cerebral spin accumulation differs even at the same PLD due to differences in blood flow 
intensity, and the SI of the brain tissue on original images differs; therefore, the signal-to-noise ratio (SNR) changes. This change influences BVA observation, but the detailed relationship remains to be clarified. Similarly, no study has investigated the relationship with PLD, which changes the apparent SNR, using a physics experiment.

In this study, we clarified differences in visualization related to blood flow intensity, vascular diameter, or PLD on ASL using a physics experiment, and examined an optimal PLD for obtaining blood flow/brain parenchyma contrast that facilitates the detection of BVA.

\section{Principle of ASL}

ASL is an imaging technique to obtain perfusion images in the absence of contrast enhancement using MRI. In this procedure, blood is magnetically labeled with radiofrequency $(\mathrm{RF})$ in the cervical region and used as an intrinsic tracer to examine intracerebral distribution after a specific time (Fig. 1).

The waiting time from spin labeling until perfusion is expressed as PLD. A satisfactory PLD in adults with brain disease is reportedly approximately 1.5 seconds. However, aging-related delay in the interval until blood flow reaches the brain tissue prolongs the PLD for obtaining normal perfusion images; therefore, imaging must be performed, establishing the PLD as 2 or 2.5 seconds. ${ }^{9)}$

Based on the original images obtained on ASL, a cerebral blood flow (CBF) map is prepared through backgroundsignal-suppression treatment and $\mathrm{CBF}$ calculation. ${ }^{10)}$ For CBF signal changes in the brain, a compartment model is applied, ${ }^{11)}$ but changes in spin signals depend on the T1 relaxation of protons. ${ }^{9)}$ In experimental systems, cerebral circulation is not considered, and, as a signal formula, a sequence-dependent, fast-spin-echo-based theoretical formula (1) is used. ${ }^{12,13)}$

$$
\text { Signal }=\mathrm{PD} \times\left(1-\mathrm{e}^{(-\mathrm{TR} / \mathrm{T} 1)}\right) \times \mathrm{e}^{(-\mathrm{TE} / \mathrm{T} 2)}
$$

In the above formula, TR refers to the repetition time, TE refers to the echo time, and PD refers to proton density.

\section{Subjects and Methods}

\section{Blood flow simulation method using a phantom and imaging conditions}

As a phantom for this verification, three extension tubes measuring $100 \mathrm{~cm}$ in length were used to measure ASL signals (inner diameter: $2.2 \mathrm{~mm}$ [Nipro, Osaka, Japan], inner diameter: $1.9 \mathrm{~mm}$ [Medikit, Tokyo, Japan], and inner diam-

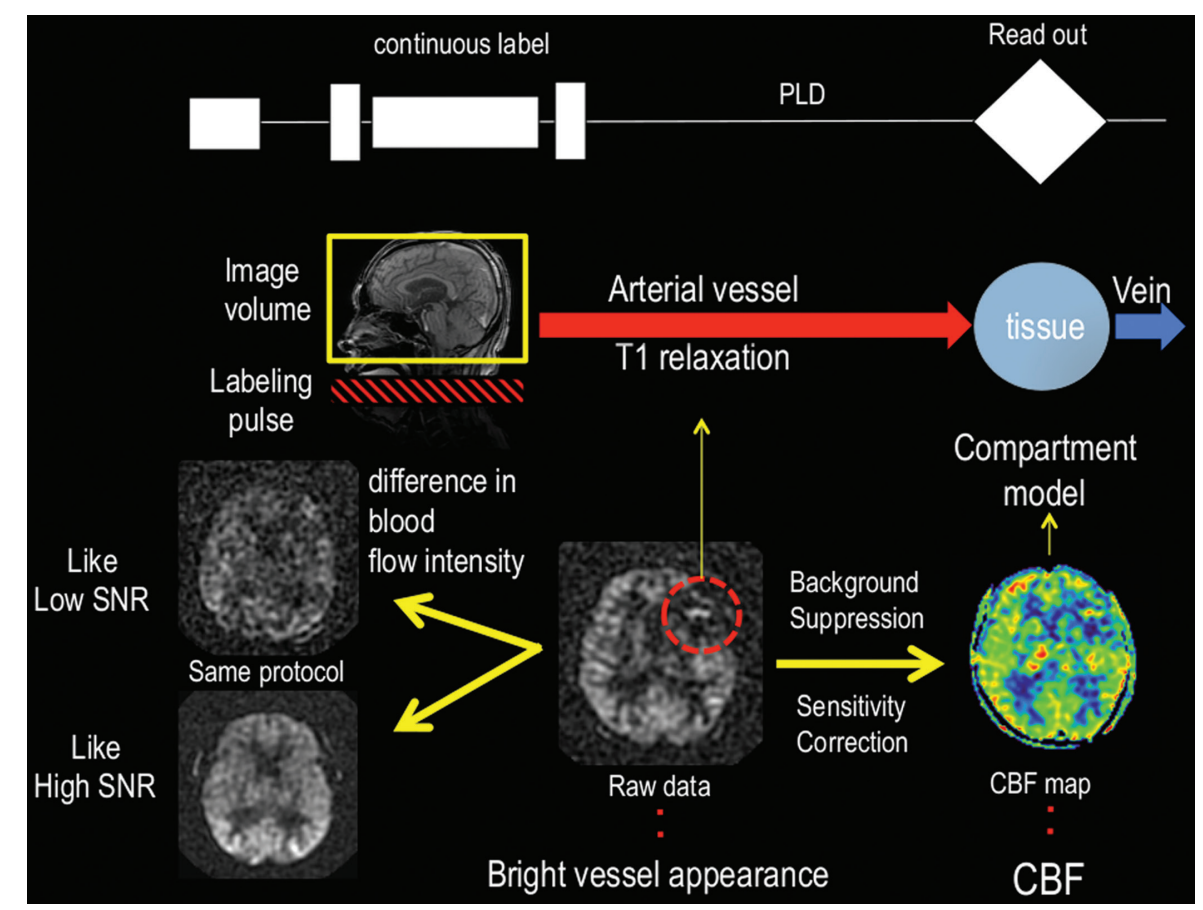

Fig. 1 ASL overview. ASL applies labeling pulse and then collects signals after the PLD to obtain perfusion images. The state of original images differs depending on the intensity of blood flow in each patient. If signals are collected with delayed perfusion to brain tissue, spin remains in the blood vessel and results in BVA. ASL: arterial spin labeling; BVA: bright vessel appearance; PLD: post-labeling delay 


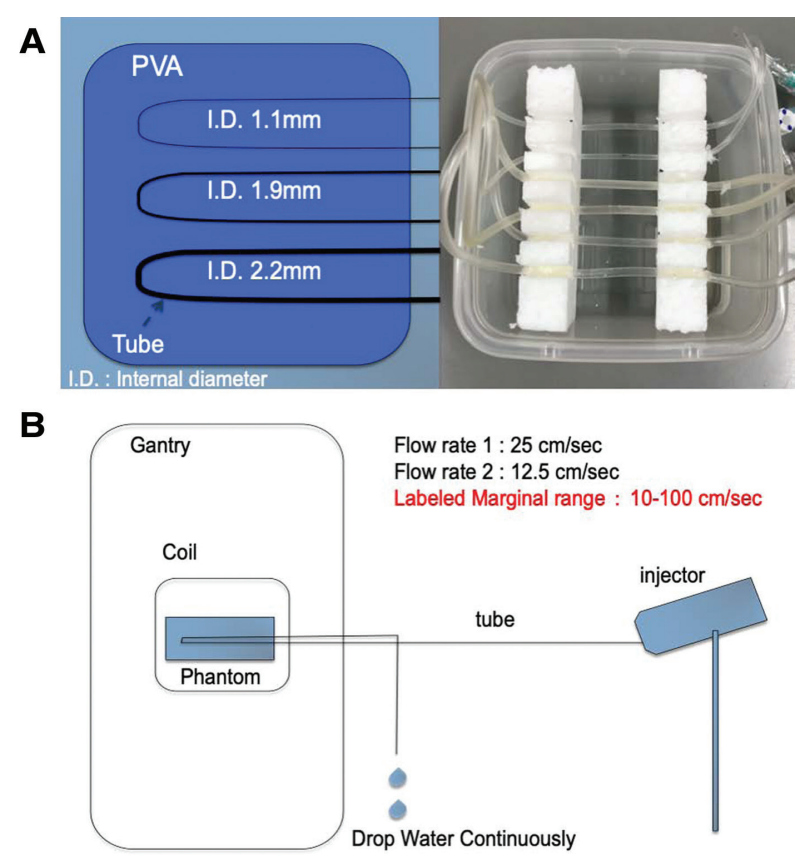

Fig. 2 (A) Phantom and (B) experimental system. (A) Plastic containers have three different inner diameters. Fill the surrounding area with PVA gel. (B) Arrangement of MRI, phantom, and injector in the experimental system. Run two types of simulated blood flow using tap water. PVA: polyvinyl alcohol

eter: $1.1 \mathrm{~mm}$ [Nipro, Osaka, Japan]). These tubes were fixed in the $U$ shape using styrofoam in a square plastic container measuring $12 \mathrm{~cm} \times 12 \mathrm{~cm} \times 4.8 \mathrm{~cm}$ such that a portion of each tube was parallel at the center of the container and their empty space was filled with polyvinyl alcohol (PVA) gel.

For blood flow simulation, the tube end was connected with an injector and tap water was infused. Tap water was placed in a $60-\mathrm{mL}$ syringe and continuously infused using the drip function of the injector during imaging (Fig. 2). The rate of infusion was established as $25 \mathrm{~cm} / \mathrm{sec}$ by setting the infusion pressure as $1 \mathrm{~mL} / \mathrm{sec}$ using a $4-\mathrm{mL}$ tube measuring $100 \mathrm{~cm}$ in length, and as $12.5 \mathrm{~cm} / \mathrm{sec}$ by setting the infusion pressure as $0.5 \mathrm{~mL} / \mathrm{sec}$. The two rates of infusion were fixed, assuming a low flow velocity in the range of flow velocity at which labeling efficiency is assured: $10-100 \mathrm{~cm} / \mathrm{sec}^{12)}$

On imaging, axial plain of the tube course at the center of the container was adopted, and the extent of imaging exceeded the size of the container. ASL was performed while changing PLD with the diameter of each tube to each rate of infusion.

Imaging conditions were established as follows: 3D ASL (pCASL), PLD: 1,025, 1,525, 2,025, 2,525, 3,025 msec, field of view: $240 \mathrm{~mm}$, slice thickness: $4.0 \mathrm{~mm}$, repetition time: Auto (4948 $\mathrm{msec}$ ), echo time: $10.5 \mathrm{msec}$, points: 512 , arms: 8 , number of signals averaged: 2 , and band width: $62.5 \mathrm{kHz}$.

The following devices were used: MRI: Signa Explorer 1.5T ver.25.1, 16-channel NV Head coil (GE Healthcare Japan, Tokyo, Japan), and Injector: Sonic Shot 7 (Nemoto Kyorindo, Tokyo, Japan).

\section{SI-measuring method and definition of $\mathrm{SI}_{\text {blood }}$ and $\mathrm{SI}_{\text {tissue }}$}

On the original images obtained on ASL, the regions of interest (ROIs) were set at 6 signal points "cephalic/central/caudal in the outflow/inflow directions," and the mean value of 3 points on the side of stronger SI in the outflow/ inflow directions was calculated. We measured the SI by randomizing the order of measurement when the flow velocity and vascular diameter were clear. At each PLD, the SI was measured/calculated twice on different days, and the mean value was regarded as SI (Fig. 3).

Tap water was placed for 24 hours under the same environment as an MRI system was installed. The T1 value was measured using the inversion recovery method, producing a value of $727 \mathrm{msec}$.

The SI value measured was converted to the SI of blood, as described below. The T1 value of tap water was converted to that of blood $\left(\mathrm{SI}_{\text {blood }}\right)$. The T1 value used for conversion at this point was $1400 \mathrm{msec}$. The process of obtaining a conversion formula (2) is shown below:

$\mathrm{SI}_{\text {blood: }}$ : SI after correction, $\mathrm{SI}_{\mathrm{b}}$ : Theoretical SI of blood obtained using Formula (1), $\mathrm{SI}_{\mathrm{w}}$ : Theoretical SI of tap water obtained using Formula (1), SI: Value measured based on the ROI in the experiment, $\mathrm{PD}_{\mathrm{b}}$ : Proton density of blood, $\mathrm{PD}_{\mathrm{w}}$ : Proton density of tap water

$$
\begin{aligned}
& \mathrm{SI}_{\text {blood }}=\left(\mathrm{SI}_{\mathrm{b}} / \mathrm{SI}_{\mathrm{w}}\right) \times \mathrm{SI} \\
& =\frac{\left\{\mathrm{PD}_{\mathrm{b}} \times\left(1-\mathrm{e}^{(-\mathrm{TR} / 1400)}\right) \times \mathrm{e}^{(-\mathrm{TE} / \mathrm{T2b})}\right\}}{\left\{\mathrm{PD}_{\mathrm{w}} \times\left(1-\mathrm{e}^{(-\mathrm{TR} / \mathrm{T} \text { value of tap water })}\right) \times \mathrm{e}^{(-\mathrm{TE} / \mathrm{T} 2 \mathrm{w})}\right\} \times \mathrm{SI}}
\end{aligned}
$$

The TE is markedly small and its influence can be ignored. In addition, the difference in proton density between the two materials is small, and there may be no marked influence.

$$
\begin{aligned}
& \fallingdotseq \frac{\left(\left(1-\mathrm{e}^{(-\mathrm{TR} / 1400)}\right)\right.}{\left.\left(1-\mathrm{e}^{(-\mathrm{TR} / \mathrm{T} 1 \text { value of tap water })}\right)\right)} \times \mathrm{SI} \\
& \mathrm{SI}_{\text {blood }}=\frac{\mathrm{SI} \times\left(\left(1-\mathrm{e}^{(-\mathrm{TR} / 1400)}\right)\right.}{\left.\left(1-\mathrm{e}^{(-\mathrm{TR} / \mathrm{T} 1 \text { value of tap water })}\right)\right)}
\end{aligned}
$$

Furthermore, the SI of the tissue on original ASL images on which normal perfusion was obtained among clinical 

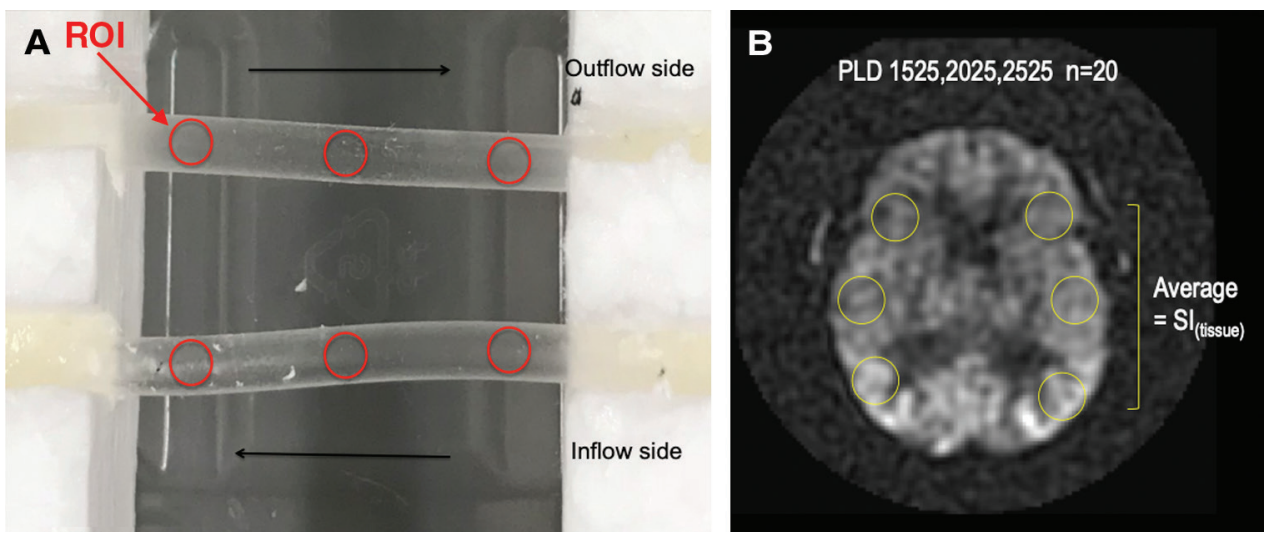

Fig. 3 Placement of the region of interest. (A) When measuring the signal on the tube, ROls are placed at six points, with three points each on the outflow side and the inflow side. (B) The average value is calculated by measuring brain tissue at six points, including left, right, back, middle, and forward, in an image in which contrast enabling normal perfusion to be observed is obtained. ROIs: regions of interest
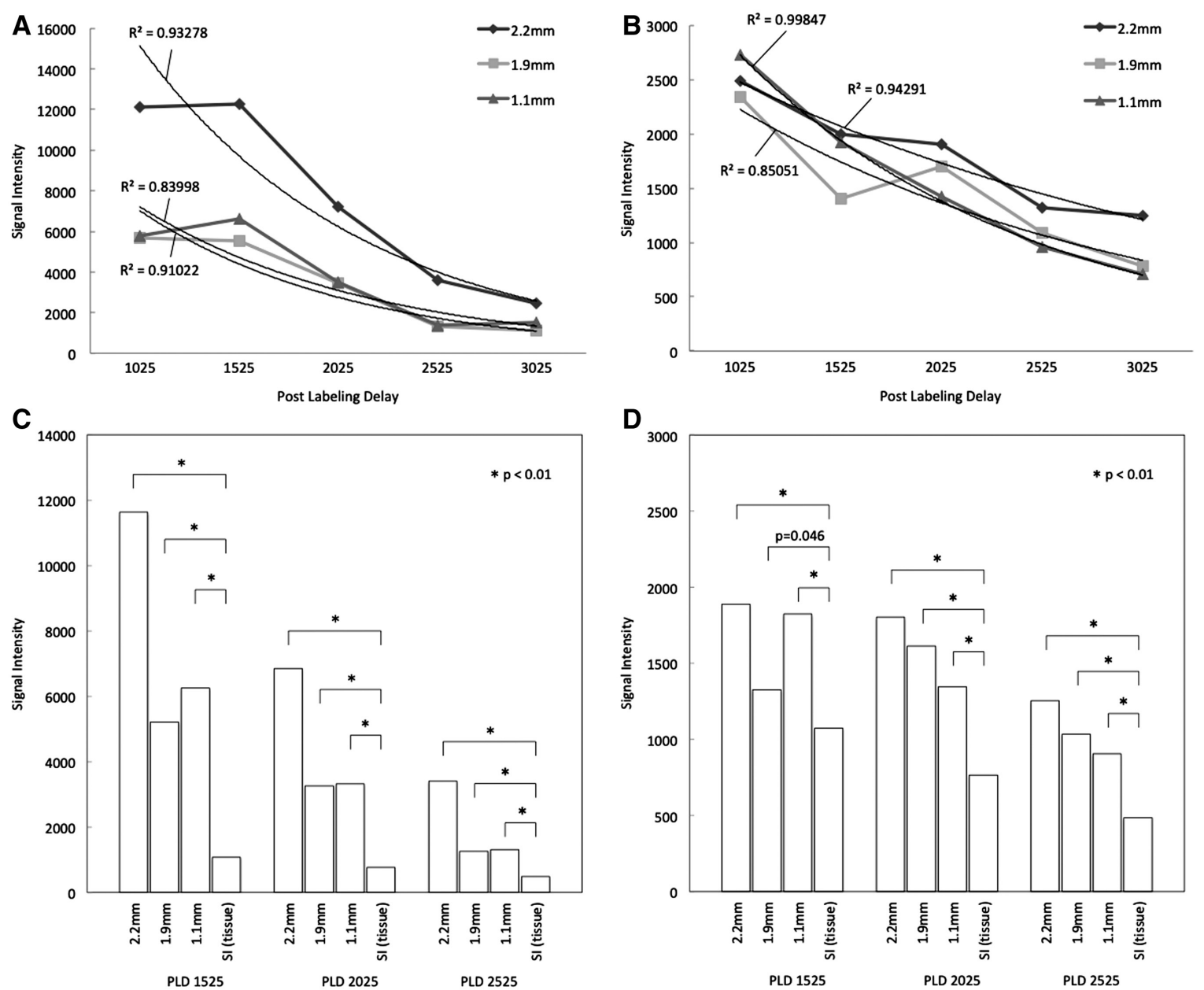

Fig. 4 Graphs of SI and $\mathrm{SI}_{\text {blood }}$ and $\mathrm{SI}_{\text {tissue. }}$ (A) $\mathrm{SI}$ in $25 \mathrm{~cm} / \mathrm{sec}$. (B) $\mathrm{SI}$ in $12.5 \mathrm{~cm} / \mathrm{sec}$. (C) Relationship of $\mathrm{SI}_{\text {blood }}$ with the $\mathrm{SI}_{\text {tissue }}$ in $25 \mathrm{~cm} / \mathrm{sec}$. (D) Relationship of $\mathrm{SI}_{\text {blood }}$ with the $\mathrm{SI}_{\text {tissue }}$ in $12.5 \mathrm{~cm} / \mathrm{sec}$. Behavior of T1 palliation is shown in $\mathbf{A}$ and $\mathbf{B}$. As shown in graphs $\mathbf{C}$ and $\mathbf{D}$, BVA can be observed because the value of $\mathrm{SI}_{\text {blood }}$ is significantly higher than that of $\mathrm{SI}_{\text {tissue. }}$. The spin signal and the background signal approach each other according to prolongation of PLD, and the spin signal value decreases at a low flow rate. BVA: bright vessel appearance; PLD: post-labeling delay; SI: signal intensity 
images was measured, and defined as the SI of the cerebral parenchyma, as described below. At the level of the basal ganglia, the SI was measured at 6 points "anterior/central/ posterior on the left and right sides," and the mean value was calculated. These values were randomly measured in 20 patients each at three PLDs of 1525, 2025, and $2525 \mathrm{msec}$, and the mean value was regarded as $\mathrm{SI}_{\text {tissue }}$ and used as a cutoff value for isolation from the brain tissue. As PLDs of 1025 and $3025 \mathrm{msec}$ are insufficient for tissue perfusion in adults, ${ }^{14)}$ patients with these values were excluded.

\section{Verification method and statistical analysis}

For verification, the SI obtained on ASL at two simulated blood flow velocities was compared among the three tubes at five PLDs. Furthermore, the $\mathrm{SI}_{\text {blood }}$ and $\mathrm{SI}_{\text {tissue }}$ were compared among the three tubes at three PLDs at two simulated blood flow velocities, assuming blood vessel-cerebral parenchyma signal contrast on clinical images.

For statistical analysis, we used the open-source free software R. The r-squared value by exponential approximation of the SI value was calculated. To compare the $\mathrm{SI}_{\text {blood }}$ and $\mathrm{SI}_{\text {tissue }}$, Wilcoxon's signed-rank test was used and significance was tested.

\section{Results}

Graphs of the SI, $\mathrm{SI}_{\text {tissue }}$, and $\mathrm{SI}_{\text {blood }}$ are shown (Fig. 4). The SI demonstrated attenuation approximate to an exponent, $\mathrm{T} 1$ relaxation.

In all tubes, the SI at $12.5 \mathrm{~cm} / \mathrm{sec}$ was lower than that at $25 \mathrm{~cm} / \mathrm{sec}$, and it decreased according to the prolongation of PLD. When the inner diameter of a tube was thin, there was a slight decrease in the SI. Furthermore, the $\mathrm{SI}_{\text {tissue }}$ decreased according to the prolongation of PLD. The $\mathrm{SI}_{\text {blood }}$ significantly exceeded the $\mathrm{SI}_{\text {tissue }}$ at 12.5 and $25 \mathrm{~cm} / \mathrm{sec}$. Furthermore, the difference between the $\mathrm{SI}_{\text {tissue }}$ and $\mathrm{SI}_{\text {blood }}$ decreased according to the prolongation of PLD at $25 \mathrm{~cm} /$ $\mathrm{sec}$ in comparison with that at $12.5 \mathrm{~cm} / \mathrm{sec}$.

\section{Discussion}

When PLD was prolonged, the SI decreased, suggesting that spin signals become similar to background noise when PLD is prolonged and that spin signals decrease when the vascular diameter is thin.

On ASL at the situation of AIS, the affected side is visualized as hypoperfusion, and spins remaining in blood vessels in the region have a high contrast with the periphery. ${ }^{8,9)}$
However, the SI of the brain tissue in which perfusion is maintained increases in comparison with the state of hypoperfusion depending on the degree of collateral pathway development or site of occlusion. When the collateral pathway involves an area adjacent to the above brain tissue, the SI of the brain tissue is approximate to the intravascular SI during spin observation on ASL, in which spatial resolution is low; it may be impossible to differentiate the former from the latter. We changed the PLD at each blood flow velocity and found that it is possible to observe spins while differentiating them from the brain tissue. The SI at $25 \mathrm{~cm} / \mathrm{sec}$ was higher than that at $12.5 \mathrm{~cm} / \mathrm{sec}$. This was possibly because the absolute volume of magnetized spins increased. Furthermore, when the flow velocity was relatively fast, the difference between the $\mathrm{SI}_{\text {tissue }}$ and $\mathrm{SI}_{\text {blood }}$ decreased according to the prolongation of PLD, suggesting that the prolongation of PLD makes spin observation difficult. When the flow velocity was slow, the SI of spins was approximate to that of the tissue at all PLDs, making differentiation difficult.

On time-of-flight magnetic resonance angiography (MRA), blood flow remaining in the scanning range for a long interval, such as retrograde or slow blood flow, may not be visualized. ${ }^{15,16)}$ Therefore, this procedure may not be appropriate for visualizing leptomeningeal anastomosis, which reflects a collateral pathway. On the other hand, ASL may facilitate observation of a collateral pathway in the acute phase. ${ }^{17-19)}$ Furthermore, there is no dependency on blood flow direction based on the principle of the imaging procedure and the SI of the whole brain can be visualized. Thus, this study suggested that it is possible to observe signals, which may be useful for the assessment of collateral pathways in AIS patients.

In this study, the SI of the brain tissue at PLDs of 1525 , 2025 , and $2525 \mathrm{msec}$, at which a contrast facilitating observation of normal perfusion was obtained, was compared with that of spins. The latter was significantly higher, suggesting that BVA observation is possible. Thus, collateral pathway spins may be recognized as high SI even on slices of the perfusion-maintained tissue adjacent to a collateral pathway in the case of AIS. Furthermore, spin signals became approximate to background signals according to the prolongation of PLD and the SI of spins decreased at a slow flow velocity, suggesting that BVA should be carefully examined in the presence of PLD prolongation or slow blood flow. In addition, as images are compared with normal perfusion images, it is necessary to select a patientfactor-matched optimal PLD for imaging in clinical 
practice. If these are applied, ASL may facilitate the assessment of a collateral pathway that cannot be observed on MRA.

As a limitation, this was a physics experiment and spin signals do not reflect the SI on clinical images. Furthermore, the SI of the background cerebral parenchyma was not compared with that of simulated blood vessels in the same experimental system; therefore, the direct signal ratio was unable to be examined. In addition, simulated blood flow was steady, differing from pulsating flow in vivo. Signals may be averaged by the length of the imaging time, but the results cannot be compared using the experiment. Results similar to those in clinical practice may be obtained by matching the simulated blood flow velocity to the accurate velocity of a collateral pathway.

\section{Conclusion}

This study suggested that ASL, in which a PLD for obtaining normal perfusion was established, facilitates BVA observation on original images at PLDs of 1525, 2025, and $2525 \mathrm{msec}$. The optimal PLD-established ASL during MRI facilitated observation of a collateral pathway in patients with AIS.

\section{Acknowledgments}

We thank the staff of the Department of Radiology, Municipal Kakunodate General Hospital for their cooperation. We also thank the staff of the Wilhelm Camp (Tohoku Section of the Japanese Society of Radiological Technology) for their advice.

\section{Disclosure Statement}

We declare no conflicts of interest.

\section{References}

1) Nogueira RG, Jadhav AP, Haussen DC, et al: Thrombectomy 6 to 24 hours after stroke with a mismatch between deficit and infarct. N Engl J Med 2018; 378: 11-21.

2) Albers GW, Marks MP, Kemp S, et al: Thrombectomy for stroke at 6 to 16 hours with selection by perfusion imaging. N Engl J Med 2018; 378: 708-718.

3) Wang DJ, Alger JR, Qiao JX, et al: The value of arterial spin-labeled perfusion imaging in acute ischemic stroke: comparison with dynamic susceptibility contrast-enhanced MRI. Stroke 2012; 43: 1018-1024.
4) Bivard A, Krishnamurthy V, Stanwell P, et al: Arterial spin labeling versus bolus-tracking perfusion in hyperacute stroke. Stroke 2014; 45: 127-133.

5) Hernandez DA, Bokkers RP, Mirasol RV, et al: Pseudocontinuous arterial spin labeling quantifies relative cerebral blood flow in acute stroke. Stroke 2012; 43: 753-758.

6) Zaharchuk G, Do HM, Marks MP, et al: Arterial spin-labeling MRI can identify the presence and intensity of collateral perfusion in patients with moyamoya disease. Stroke 2011; 42: 2485-2491.

7) Kronenburg A, Bulder MMM, Bokkers RPH, et al: Cerebrovascular reactivity measured with ASL perfusion MRI, ivy sign, and regional tissue vascularization in moyamoya. World Neurosurg 2019; 125: e639-e650

8) Yoo RE, Yun TJ, Rhim JH, et al: Bright vessel appearance on arterial spin labeling MRI for localizing arterial occlusion in acute ischemic stroke. Stroke 2015; 46: 564-567.

9) Fujiwara Y, Kimura H: MR perfusion imaging using arterial spin labeling. Med Info Sci 2015; 32: 37-41.

10) Garcia DM, Duhamel G, Alsop DC: Efficiency of inversion pulses for background suppressed arterial spin labeling. Magn Reson Med 2005; 54: 366-372.

11) Alsop DC, Detre JA: Reduced transit-time sensitivity in noninvasive magnetic resonance imaging of human cerebral blood flow. J Cereb Blood Flow Metab 1996; 16: 1236-1249.

12) Dai W, Garcia D, de Bazelaire C, et al: Continuous flowdriven inversion for arterial spin labeling using pulsed radio frequency and gradient fields. Magn Reson Med 2008; 60: 1488-1497.

13) Busse RF, Riederer SJ, Fletcher JG, et al: Interactive fast spin-echo imaging. Magn Reson Med 2000; 44: 339-348.

14) Alsop DC, Detre JA, Golay X, et al: Recommended implementation of arterial spin-labeled perfusion MRI for clinical applications: a consensus of the ISMRM perfusion study group and the European consortium for ASL in dementia. Magn Reson Med 2015; 73: 102-116.

15) Kasai T, Doi T: Imaging Technology for Magnetic Resonance. Ohmsha. Tokyo, 2001, 140-142.

16) Hashemi RH, Bradley WG, Lisanti CJ: MRIThe Basics, Second edition. Medical Sciences International. Tokyo, 2004, 324-336.

17) Zaharchuk G: Arterial spin-labeled perfusion imaging in acute ischemic stroke. Stroke 2014; 45: 1202-1207.

18) Bulder MM, Bokkers RP, Hendrikse J, et al: Arterial spin labeling perfusion MRI in children and young adults with previous ischemic stroke and unilateral intracranial arteriopathy. Cerebrovasc Dis 2014; 37: 14-21.

19) Petersen ET, Zimine I, Ho YC, et al: Non-invasive measurement of perfusion: a critical review of arterial spin labelling techniques. Br J Radiol 2006; 79: 688-701. 